\title{
On the Cook-Kuroda Criterion in Scattering Theory`
}

\author{
Tosio Kato \\ Department of Mathematics, University of California, Berkeley, California 94720, USA
}

\begin{abstract}
A new criterion of the Cook-Kuroda type for the existence of the wave operator in the two-space scattering theory is introduced. The condition is quite simple, but it generalizes not only the original Cook-Kuroda condition but also its generalization recently given by Schechter. Specialized to the onespace case, it is actually equivalent to Schechter's condition for an optimal choice of factorization. An application to potential scattering leads to a new result.
\end{abstract}

\section{Introduction}

Recently Schechter [1] and Simon [2] generalized the 20-year-old Cook-Kuroda criterion $[3,4]$ for the existence of the wave operator in scattering theory. The purpose of the present paper is to contribute another generalization in the context of two-space scattering theory [5]. Our condition (Theorem I) has several advantages. First, it is formally simpler than others [1-4], involving only bounded operators. Second, it has a simple, purely time-dependent proof. Third, it is valid in the two-space setting without any extra assumptions on the identification operator $J$ except that $J$ is bounded. Fourth, Schechter's theorem can easily be reduced to ours, so that our results contain a simplified proof of a two-space version of his theorem. At the same time, this shows that our result is in general stronger than Schechter's.

On the other hand, Schechter's condition is extremely flexible, involving a (formal) factorization of the perturbation that can be chosen in many different ways. In fact we shall show that some favorable choices of the factorization lead to a result equivalent to ours (Theorem III).

Let us first state our theorems. In two-space scattering theory, one considers two selfadjoint operators $H_{j}, j=1,2$, each acting in its Hilbert space $\mathfrak{H}_{j}$, and a bounded linear operator $J$ (the identification operator) on $\mathfrak{H}_{1}$ to $\mathfrak{H}_{2}$. We denote by $U_{j}(t)=\exp \left(-i t H_{j}\right)$ the unitary group generated by $-i H_{j}$. The associated wave

* This work was partially supported by NSF Grant MCS 76-04655 
operator $W_{+}=W_{+}\left(H_{2}, H_{1} ; J\right)$ will be defined by

$$
W_{+} f=\lim _{t \rightarrow \infty} W(t) f, \quad W(t)=U_{2}(-t) J U_{1}(t),
$$

whenever the limit exists. Obviously the domain $\mathfrak{D}\left(W_{+}\right)$of $W_{+}$is a (closed) subspace of $\mathfrak{H}_{1}$. In this paper we do not extend $W_{+}$beyond this domain. Another wave operator $W_{-}$defined with $-\infty$ instead of $\infty$ in (1.1) can be handled in the same way.

Denoting by $R_{j}(z)=\left(H_{j}-z\right)^{-1}$ the resolvent, and by $\varrho\left(H_{j}\right)$ the resolvent set, of $H_{j}$, we set

$$
C(z)=R_{2}(z) J-J R_{1}(z), \quad z \in \varrho \equiv \varrho\left(H_{1}\right) \cap \varrho\left(H_{2}\right) .
$$

Our main result now reads

Theorem I. Let $z \in \varrho$. If $f \in \mathfrak{H}_{1}$ satisfies

$$
\int_{0}^{\infty}\left\|C(z) U_{1}(t) f\right\| d t<\infty
$$

then $f \in \mathfrak{D}\left(W_{+}\right)$.

It is convenient to state Theorem I in a different form by introducing the set $\mathfrak{M}(z)$ of all $f \in \mathfrak{H}_{1}$ satisfying (1.3). $\mathfrak{M}(z)$ is obviously a linear manifold in $\mathfrak{H}_{1}$. Let us denote its closure by $[\mathfrak{M}(z)]$. Then Theorem I is equivalent to

Theorem I'. $[\mathfrak{M}(z)] \subset \mathcal{D}\left(W_{+}\right)$.

Regarding the dependence of $\mathfrak{M}(z)$ and $[\mathfrak{M}(z)]$ on $z$, we have

Theorem $\mathbf{I}^{\prime \prime}$. For $\operatorname{Im} z>0, \mathfrak{M}(z)=\mathfrak{M}$ is independent of $z$, while $\mathfrak{M}(z) \subset \mathfrak{M}$ for $\operatorname{Im} z<0$. $[\mathfrak{M}(z)]=[\mathfrak{M}]$ is independent of $z \in \varrho$.

We shall prove Theorem I' in Sect. 2, and study its relationship to other criteria of the Cook-Kuroda type, in particular Schechter's, in Sects. 3 and 4. Theorem I" will be proved also in Sect. 4. These sections contain other results related to the Schechter factorization. Sect. 5 contains an application to potential scattering in $R^{3}$.

\section{Proof of Theorem $I^{\prime}$}

First we note some obvious facts regarding $\mathfrak{M}(z)$ and $[\mathfrak{M}(z)]$. Since $f$ satisfies $(1.3)$ if and only if $U_{1}(s) f$ does, where $s$ is any real number, $\mathfrak{M}(z)$ is invariant under the map $U_{1}(s)$. Hence the same is true of $[\mathfrak{M}(z)]$, which therefore reduces $H_{1}$. This implies, in particular, that $R_{1}\left(z^{\prime}\right) \mathfrak{M}(z)$ is a dense subset of $[\mathfrak{M}(z)]$ for any $z^{\prime} \in \varrho\left(H_{1}\right)$.

A simple calculation gives (we write $R_{j}(z)=R_{j}, C(z)=C$ for simplicity)

$$
(d / d t)\left(R_{2} W(t) R_{1} f, g\right)=-i\left(U_{2}(-t) C U_{1}(t) f, g\right)
$$

for any $f \in \mathfrak{H}_{1}$ and $g \in \mathfrak{H}_{2}$. Hence for $t^{\prime}<t^{\prime \prime}$

$$
\left\|R_{2} W\left(t^{\prime \prime}\right) R_{1} f-R_{2} W\left(t^{\prime}\right) R_{1} f\right\| \leqq \int_{t^{\prime}}^{t^{\prime \prime}}\left\|C U_{1}(t) f\right\| d t .
$$

Assume now that $f \in \mathfrak{M}(z)$ so that (1.3) is true. Then (2.1) shows that $\lim R_{2} W(t) R_{1} f$ exists. (Here and in what follows lim refers to $t \rightarrow \infty$.) Since $R_{2} W(t)$ is uniformly bounded in $t$ and $R_{1} \mathfrak{M}(z)$ is dense in $[\mathfrak{M}(z)]$ as noted above, it follows 
that $s-\lim R_{2} W(t)$ exists on $[\mathfrak{M}(z)]$. Then Lemma 2.1 given below shows that $s$ - $\lim W(t) R_{1}$ exists on $[\mathfrak{M}(z)]$. Since $W(t)$ is uniformly bounded, a similar argument shows that $s-\lim W(t)$ exists on $[\mathfrak{M}(z)]$. This means that $[\mathfrak{M}(z)] \subset \mathfrak{D}\left(W_{+}\right)$.

Lemma 2.1. $s-\lim \left[R_{2}(z) W(t)-W(t) R_{1}(z)\right]=0$ on $[\mathfrak{M}(z)]$.

Proof. Let $\phi \in C_{0}^{\infty}(-\infty, \infty)$ and set

$$
g=\int_{-\infty}^{\infty} \phi(s) U_{1}(s) f d s, \quad f \in \mathfrak{M}(z)
$$

The set of all such $g$ 's is dense in $\mathfrak{M}(z)$, hence in $[\mathfrak{M}(z)]$ too, since (2.2) tends to $f$ if $\phi$ tends to the delta function. On the other hand, we have

$$
\left\|C U_{1}(t) g\right\| \leqq \int_{-\infty}^{\infty}|\phi(s)|\left\|C U_{1}(t+s) f\right\| d s \quad(C=C(z)) .
$$

Since $\left\|C U_{1}(t) f\right\|$ is integrable in $t \in(0, \infty)$ and since $\phi$ has compact support, it follows that $\lim \left\|C U_{1}(t) g\right\|=0$. Since $C U_{1}(t)$ is uniformly bounded and the $g$ 's are dense in $[\mathfrak{M}(z)]$ as noted above, we conclude that $s-\lim C U_{1}(t)=0$ on $[\mathfrak{M}(z)]$. The lemma then follows from the identity

$$
R_{2} W(t)-W(t) R_{1}=U_{2}(-t) C U_{1}(t) .
$$

\section{Relation to Schechter's Theorem}

We now compare our results with other criteria of the Cook-Kuroda type [1-4]. Since Schechter's theorem [1] is the strongest one among them, it suffices to consider it.

Schechter's condition involves a "factorization" of the perturbation which, in the context of two-space theory, takes the form

$$
\left(J u, H_{2} v\right)-\left(J H_{1} u, v\right)=(A u, B v),
$$

assumed to be true for every $u \in \mathfrak{D}\left(H_{1}\right)$ and $v \in \mathfrak{D}\left(H_{2}\right)$. Here $A$ is a linear operator from $\mathfrak{H}_{1}$ to a Banach space $\mathfrak{S}$ with $\mathfrak{D}(A) \supset \mathfrak{D}\left(H_{1}\right)$, and $B$ is a linear operator from $\mathfrak{H}_{2}$ to $\mathfrak{K}^{*}$ (the adjoint space of $\mathfrak{R}$ ) with $\mathfrak{D}(B) \supset \mathfrak{D}\left(H_{2}\right)$. $B$ is assumed to be $\mathrm{H}_{2}$-bounded.

Any operator $A$ that appears in this Schechter factorization (together with some $B$ ) will be called a Schechter operator (for the triplet $\left\{H_{2}, H_{1}, J\right\}$ ).

Schechter's theorem now reads, with a slight generalization,

Theorem II. Let $A$ be a Schechter operator. If $f \in \mathfrak{D}\left(H_{1}\right)$ satisfies the condition

$$
\int_{t_{f}}^{\infty}\left\|A U_{1}(t) f\right\| d t<\infty
$$

for some real number $t_{f}$, then $f \in \mathfrak{D}\left(W_{+}\right)$.

Remark 3.1. (a) In [1] the condition $f \in \mathfrak{D}\left(H_{1}\right)$ is assumed, though not stated explicitly.

(b) Even for $f \in \mathfrak{D}\left(H_{1}\right),\left\|A U_{1}(t) f\right\|$ may not be measurable in $t$, since $A$ is not assumed to be $H_{1}$-bounded or closable. Thus the integral in (3.2) should be taken in the sense of an upper integral. 
(c) In [1] only the single-space case $\left(\mathfrak{H}_{1}=\mathfrak{H}_{2}, J=1\right)$ is considered. In a lecture at the Utah Conference (July 1978), Schechter generalized the theorem to the twospace case under certain additional conditions on $J$. In Theorem II, however, we need no extra conditions on $J$.

As before, it is convenient to rewrite Theorem II by introducing the set $\mathfrak{M}(A)$ of all $f \in \mathfrak{D}\left(H_{1}\right)$ satisfying (3.2). Again it is obvious that $\mathfrak{M}(A)$ is a linear manifold in $\mathfrak{H}_{1}$ invariant under $U_{1}(t)$, and its closure $[\mathfrak{M}(A)]$ reduces $H_{1}$. Theorem II is equivalent to

\section{Theorem II'. $[\mathfrak{M}(A)] \subset \mathfrak{D}\left(W_{+}\right)$.}

We shall show that Theorem II' can be reduced to Theorem I'. We achieve this by showing not $\mathfrak{M}(A) \subset \mathfrak{M}(z)$ (which is probably untrue) but $[\mathfrak{M}(A)] \subset[\mathfrak{M}(z)]$. More precisely:

Theorem III. For any Schechter operator $A$, we have $[\mathfrak{M}(A)] \subset[\mathfrak{M}]$ (where $[\mathfrak{M}]$ is the common space $[\mathfrak{M}(z)]$, see Theorem $\left.I^{\prime \prime}\right)$. On the other hand, for any triplet $\left\{H_{2}, H_{1}, J\right\}$ there are Schechter operators $A$ with $[\mathfrak{M}(A)]=[\mathfrak{M}]$.

Remark 3.2. Any triplet $\left\{H_{2}, H_{1}, J\right\}$ has infinitely many Schechter factorizations. A simple and useful one is given by

$$
A=A(z)=C(z)\left(H_{1}-z\right), \quad B=B(z)=-\left(H_{2}-\bar{z}\right)
$$

where $z \in \varrho$ and $C(z)$ is as before (1.2). Here we take $\mathfrak{H}=\mathfrak{H}_{2}, \mathfrak{D}(A)=\mathfrak{D}\left(H_{1}\right)$ and, of course, $\mathfrak{D}(B)=\mathfrak{D}\left(\mathrm{H}_{2}\right)$. We shall refer to $A(z)$ as an optimal Schechter operator, since it gives the optimal result stated in Theorem III.

\section{Proof of Theorem III}

Lemma 4.1. $[\mathfrak{M}(A)] \subset[\mathfrak{M}(z)]$ for all $z \in \varrho$.

Proof. As is easily seen, (3.1) implies

$$
C=R_{2} J-J R_{1}=-\left(B R_{2}^{*}\right) * A R_{1},
$$

where we have again written $R_{j}=R_{j}(z), C=C(z)$. Here $\left(B R_{2}^{*}\right)^{*}$ is a bounded operator on $\mathfrak{R}^{* *}$ to $\mathfrak{H}_{2}$ because $B$ is $H_{2}$-bounded. Since $A$ has range in $\mathcal{K},(4.1)$ makes sense by the canonical embedding of $\Re$ in $\mathfrak{R}^{* *}$.

Let $f \in \mathfrak{M}(A)$. Then

$$
\int_{t_{f}}^{\infty}\left\|C U_{1}(t)\left(H_{1}-z\right) f\right\| d t=\int_{t_{f}}^{\infty}\left\|\left(B R_{2}^{*}\right)^{*} A U_{1}(t) f\right\| d t<\infty
$$

by (4.1) and (3.2) because $B R_{2}^{*}$ is bounded. It follows that $\left(H_{1}-z\right) f \in \mathfrak{M}(z)$; the $t_{f}$ on the left is irrelevant since $C$ is bounded. Since $[\mathfrak{M}(z)]$ reduces $H_{1}$, we conclude that $f \in[\mathfrak{M}(z)]$. This proves that $\mathfrak{M}(A) \subset[\mathfrak{M}(z)]$, and hence $[\mathfrak{M}(A)] \subset[\mathfrak{M}(z)]$ for any $z \in Q$.

Lemma 4.2. The Schechter operator $A(z)=C(z)\left(H_{1}-z\right)$ is optimal in the sense that $[\mathfrak{M}(A(z))]=\left[\mathfrak{M}\left(z^{\prime}\right)\right]$ for any $z^{\prime} \in \varrho$.

Corollary 4.3. $[\mathfrak{M}(z)]$ is independent of $z \in \varrho$.

Proof of Lemma 4.2. We have the identity

$$
C\left(z^{\prime}\right)=\left(H_{2}-z\right) R_{2}\left(z^{\prime}\right) C(z)\left(H_{1}-z\right) R_{1}\left(z^{\prime}\right), \quad z, z^{\prime} \in \varrho .
$$


Hence, writing $A=A(z)$,

$$
\begin{aligned}
\left\|A U_{1}(t) R_{1}\left(z^{\prime}\right) f\right\| & =\left\|C(z)\left(H_{1}-z\right) R_{1}\left(z^{\prime}\right) U_{1}(t) f\right\| \\
& \leqq\left\|\left(H_{2}-z^{\prime}\right) R_{2}(z)\right\|\left\|C\left(z^{\prime}\right) U_{1}(t) f\right\|, \quad f \in \mathfrak{H}_{1} .
\end{aligned}
$$

Since $\left\|\left(H_{2}-z^{\prime}\right) R_{2}(z)\right\|<\infty$, this inequality shows that $f \in \mathfrak{M}\left(z^{\prime}\right)$ implies $R_{1}\left(z^{\prime}\right) f \in \mathfrak{M}(A)$. Since $[\mathfrak{M}(A)]$ reduces $H_{1}$ as remarked above, it follows that $f \in[\mathfrak{M}(A)]$. Thus $\mathfrak{M}\left(z^{\prime}\right) \subset[\mathfrak{M}(A)]$, hence $\left[\mathfrak{M}\left(z^{\prime}\right)\right] \subset[\mathfrak{M}(A)]$. Since the opposite inclusion is known (Lemma 4.1), we have proved that $[\mathfrak{M}(A)]=\left[\mathfrak{M}\left(z^{\prime}\right)\right]$.

It remains to complete the proof of Theorem $I^{\prime \prime}$. First we prove

Lemma 4.4. Let $z, z^{\prime} \in \varrho$, with $\operatorname{Im} z^{\prime}>0$. Then $f \in \mathfrak{M}(z)$ implies $R_{1}\left(z^{\prime}\right) f \in \mathfrak{M}(z)$.

Proof. Since $R_{1}\left(z^{\prime}\right)=i \int_{0}^{\infty} \exp \left(i z^{\prime} s\right) U_{1}(s) d s$, we have for $f \in \mathfrak{M}(z)$

$$
\begin{aligned}
\int_{0}^{\infty}\left\|C(z) U_{1}(t) R_{1}\left(z^{\prime}\right) f\right\| d t & \leqq \int_{0}^{\infty} e^{-y^{\prime} s} d s \int_{0}^{\infty}\left\|C(z) U_{1}(t+s) f\right\| d t \\
& \leqq K_{f} \int_{0}^{\infty} e^{-y^{\prime} s} d s=K_{f} / y^{\prime}<\infty,
\end{aligned}
$$

where $y^{\prime}=\operatorname{Im} z^{\prime}>0$ and $K_{f}$ is the finite number (1.3). Hence $R_{1}\left(z^{\prime}\right) f \in \mathfrak{M}(z)$.

Lemma 4.5. Let $z, z^{\prime}$ be as in Lemma 4.4. Then $\mathfrak{M}(z) \subset \mathfrak{M}\left(z^{\prime}\right)$.

Proof. (4.2) gives

$$
\left\|C\left(z^{\prime}\right) U_{1}(t) f\right\| \leqq k\left\|C(z) U_{1}(t)\left[1+\left(z^{\prime}-z\right) R_{1}\left(z^{\prime}\right)\right] f\right\|,
$$

where $k=\left\|\left(H_{2}-z\right) R_{2}\left(z^{\prime}\right)\right\|<\infty$. Suppose now that $f \in \mathfrak{M}(z)$. Then $R_{1}\left(z^{\prime}\right) f \in \mathfrak{M}(z)$ by Lemma 4.4 , so that $\left[1+\left(z^{\prime}-z\right) R_{1}\left(z^{\prime}\right)\right] f \in \mathfrak{M}(z)$ too. Hence the right member of (4.3) is integrable in $t \in(0, \infty)$, and the same is true of the left member. This means that $f \in \mathfrak{M}\left(z^{\prime}\right)$.

Obviously Lemma 4.5 completes the proof of Theorem I".

\section{An Application to Potential Scattering}

Consider potential scattering in $\mathbb{R}^{3}$ :

$$
H_{1}=-\Delta, \quad H_{2}=-\Delta+V(x), \quad x \in \mathbb{R}^{3} .
$$

For simplicitly we assume that

$$
V=V_{1}+V_{2}
$$

where the $V_{j}$ are real-valued and

$$
0 \leqq V_{1} \in \mathfrak{Q}_{\text {loc }}^{1}\left(\mathbb{R}^{3}\right), \quad V_{2} \in \mathfrak{Q}^{2}\left(\mathbb{R}^{3}\right)+\mathfrak{Q}^{\infty}\left(\mathbb{R}^{3}\right) .
$$

Then $H_{1}$ and $H_{2}$ are selfadjoint in $\mathfrak{H}=\mathfrak{L}^{2}\left(\mathbb{R}^{3}\right)$. Here $H_{2}$ should be taken as the maximal realization in $\mathfrak{H}$ of the formal differential operator $-\Delta+V$ or, equivalently, as the form sum of $H_{1}+V_{2}$ and $V_{1}$, both of which are semibounded (see [6]). Note that $V_{2}$ is $H_{1}$-bounded with $H_{1}$-bound 0 .

We shall show that if in addition

$$
V_{j} \in \mathbb{Q}^{j}\left(\mathbb{R}^{3},(1+|x|)^{-k} d x\right), \quad j=1,2, \text { for some } k<1,
$$

then the wave operators $W_{ \pm}\left(H_{2}, H_{1} ; 1\right)$ exist on all of $\mathfrak{H}$. 
To this end we use the optimal Schechter operator (3.3), which should give the same result as our theorem. In our case with $J=1,(3.3)$ becomes

$$
A=R_{2}(z)\left(H_{1}-z\right)-1, \quad \mathfrak{D}(A)=\mathfrak{D}\left(H_{1}\right) .
$$

We choose $z$ real and sufficiently negative that $H_{j}-z \geqq 1$ for $j=1,2$. One might want to write $A=-R_{2}(z) V$, but this is hard to justify in general due to the singularity of $V$. But it is not difficult to show that

$$
A u=-\left(V_{1}^{1 / 2} R_{2}(z)\right)^{*} V_{1}^{1 / 2} u-R_{2}(z) V_{2} u
$$

provided $u \in \mathfrak{D}\left(H_{1}\right)$ and $V_{1}^{1 / 2} u \in \mathfrak{H}$. Note that $V_{1}^{1 / 2} R_{2}(z) \in \mathscr{B}(\mathfrak{G})$ because $H_{2}$ is the form sum of $H_{1}+V_{2}$ and $V_{1}$, and that $u \in \mathfrak{D}\left(H_{1}\right)$ implies $V_{2} u \in \mathfrak{H}$. It follows that

$$
\|A u\| \leqq \operatorname{const}\left(\left\|V_{1}^{1 / 2} u\right\|+\left\|V_{2} u\right\|\right) .
$$

Now let $f(x)=\exp \left(-|x-a|^{2} / 2\right)$ with a constant $a \in \mathbb{R}^{3}$, and $u(t)=U_{1}(t) f$ $=\exp \left(-i t H_{1}\right) f$. It is well known (see e.g. [7, pp. 536, 7]) that

$$
\left\|(1+|x|)^{k / 2} u(t)\right\|_{\mathfrak{R}_{\infty}}=O\left(t^{-(3-k) / 2}\right), \quad t \rightarrow \infty .
$$

In view of the assumption (5.4), it follows that $\left\|V_{1}^{1 / 2} u(t)\right\|$ and $\left\|V_{2} u(t)\right\|$ are $O\left(t^{-(3-k) / 2}\right)$. Hence the same is true of $\|A u(t)\|$ by (5.7), verifying condition (3.2).

Since the set of $f$ with varying $a \in \mathbb{R}^{3}$ spans a dense set in $\mathfrak{H}$, we have proved that $\mathfrak{D}\left(W_{+}\right)=\mathfrak{G}$. The same is true of $W_{-}$.

Remark 5.1. (a) A more obvious "factorization" with $A=|V|^{1 / 2}, B=(\operatorname{sign} V)|V|^{1 / 2}$ does not work, since this violates the condition $\mathfrak{D}(A) \supset \mathfrak{D}\left(H_{1}\right)$ (except when $k=0$ ).

(b) If $V_{1}=0,(5.4)$ reduces to Kuroda's condition [4].

\section{References}

1. Schechter, M.: A new criterion for scattering theory. Duke Math. J. 44, 863-872 (1977)

2. Simon, B.: Scattering theory and quadratic forms: On a theorem of Schechter. Commun. Math. Phys. 53, 151-153 (1977)

3. Cook, J.M.: Convergence to the Møller wave-matrix. J. Math. Phys. 36, 82-87 (1957)

4. Kuroda, S.T.: On the existence and the unitary property of the scattering operator. Nuovo Cimento 12, 431-454 (1959)

5. Kato, T.: Scattering theory with two Hilbert spaces. J. Funct. Anal. 1, 342-369 (1967)

6. Kato, T.: A second look at the essential selfadjointness of the Schrödinger operators. In: Physical reality and mathematical description, pp. 193-201. Dortrecht: Reidel 1974

7. Kato, T.: Perturbation theory for linear operators, 2nd ed. Berlin, Heidelberg, New York: Springer 1976

Communicated by B. Simon

Received January 9, 1979 